\title{
ARTÍCULOS
}

\section{Alcances sobre la didáctica de la expresión oral y escrita en el aula de enseñanza media}

\author{
On the didactic of oral and written production \\ implemented in Secondary Level Classrooms
}

\author{
Roberto Casanova S., Yasna Roldán V. \\ Centro de Idiomas \\ Facultad de Filosofía y Humanidades \\ Universidad Austral de Chile \\ roberto.casanova@uach.cl \\ yasnaroldan@uach.cl \\ Telf.: (56) 632293664
}

\section{RESUMEN}

El propósito de este trabajo es revisar algunas orientaciones en relación a la didáctica de la enseñanza de la oralidad y la escritura del español como lengua materna, con énfasis en su implementación en establecimientos de educación secundaria. Se actualizan los antecedentes expuestos en el artículo "Didáctica de la expresión oral y escrita en el liceo" (Burgos, 1976) del primer número de "Estudios Pedagógicos". Se complementan y discuten los puntos revisados por Burgos en cuanto el tema de la didáctica de la lengua oral formal y la escritura, tópicos que siguen teniendo una indiscutible vigencia. La enseñanza en el aula requiere una revisión o reformulación de la didáctica que aborde las competencias discursivas desde una perspectiva de género que conlleve a concebir la escritura y la oralidad en etapas de deconstrucción de las características del género, de producción guiada e individual y de revisión.

Palabras clave: habilidades discursivas, español como lengua materna, didáctica.

ABSTRACT

The purpose of this paper is to review some considerations related to the didactics of teaching speaking and writing of Spanish as L1, emphasizing its implementation in high-school classrooms. Results presented in the article "Didactics of speaking and writing in high school" (Burgos, 1976) on the first issue of Estudios Pedagógicos are updated. The results obtained by Burgos regarding the didactics of formal oral speech and writing are complemented and discussed and considered still as of absolute validity. Classroom education requires an analysis and a reformulation of its didactics incorporating the discursive abilities from a gender approach which leads to understanding writing and speaking as deconstruction stages of gender qualities, of guided and individual production and of review.

Key words: discourse abilities, Spanish L1, didactics. 


\section{INTRODUCCIÓN}

El desarrollo de competencias comunicativas en el idioma español como lengua materna es un ámbito de investigación bastante productivo en la actualidad. De ello dan cuenta manuales y textos de estudio, libros, capítulos de libros especializados, proyectos y artículos de investigación, encuentros, congresos y simposios. En el trabajo que se realiza a nivel nacional e internacional, destacan en este escenario las iniciativas generadas al interior de instituciones de educación superior, pues el dominio de estas habilidades es también un factor determinante al momento de evaluar el ingreso, la permanencia y la titulación oportuna de estudiantes al finalizar los estudios de pregrado.

En este contexto, no obstante, la educación secundaria -y, por supuesto, la enseñanza primaria- se advierte aún como un espacio en el que se requiere un trabajo mancomunado entre las instituciones de educación superior, los grupos de investigación y el Ministerio de Educación, que se traduzca en políticas públicas que permitan a estudiantes, independientemente de la dependencia administrativa del establecimiento al que estén adscritos, dominar competencias que les permitan hacer uso del lenguaje y participar de manera efectiva a través de él en distintas situaciones sociales. Como se ha señalado, existe bastante investigación a este respecto, pero no siempre es posible advertir en los establecimientos educacionales, fuera de iniciativas aisladas, y en algunos casos con una continuidad irregular, acciones concretas y de carácter nacional que propicien el desarrollo de estas competencias en todos los estudiantes. De esta forma, no debe extrañar que el resultado de alguna prueba estandarizada en el sector de Lenguaje y comunicación sea tema de la prensa y no constituya, por sobre todo, un espacio de reflexión y evaluación de lo que se está haciendo en esta materia.

El siguiente trabajo tiene por objetivo revisar algunas orientaciones en relación a la didáctica de la enseñanza de la oralidad y la escritura del español como lengua materna, con énfasis en su implementación en establecimientos de educación secundaria. Surge con la idea de actualizar, en conmemoración del aniversario número 40 de la revista "Estudios Pedagógicos", los antecedentes expuestos en uno de los artículos contenidos en el primer número de la revista: "Didáctica de la expresión oral y escrita en el liceo" (Burgos, 1976). De esta manera, sin afanes de exhaustividad teórica ni metodológica, se discuten y complementan los puntos revisados por el autor en el artículo señalado. Con todo, un hecho fundamental, es que el tema de discusión tiene hoy una indiscutible vigencia.

El trabajo se estructura en tres apartados fundamentales. El primero describe las orientaciones establecidas por el Ministerio de Educación para la enseñanza de la lengua materna en Chile. Luego, se analizan algunas orientaciones para implementar la didáctica de la enseñanza de la oralidad, por una parte, y la enseñanza de la escritura, por otra parte, ambas en el contexto de la enseñanza media. Finalmente, se esbozan algunas conclusiones en torno a esta revisión.

\section{LA ENSEÑANZA DEL LENGUAJE, ORIENTACIONES DESDE EL CURRÍCULUM ESCOLAR}

Los lineamientos de la enseñanza de las distintas disciplinas enmarcadas en el currículum escolar de la enseñanza media tienen sus antecedentes en el Decreto Supremo de Educación $\mathrm{N}^{\circ} 254$, marco en el que se propuso un ajuste curricular cuya implementación 
se realizó gradualmente a partir del año 2010 y hasta el año 2013, implementando de esta manera las modificaciones tanto para la Formación General como para la Formación Diferenciada Técnico Profesional (Ministerio de Educación [Mineduc], 2009). Para el caso de la asignatura cuyo propósito es la enseñanza de la lengua materna, se propuso, además, la modificación de su nombre: pasó de llamarse Lengua Castellana y Comunicación, a denominarse Sector de Lenguaje y comunicación.

El sector de Lenguaje y comunicación, de acuerdo al Ministerio de Educación, tiene como propósito el desarrollo de competencias comunicativas para el desarrollo integral de los estudiantes, propuesta formativa que se articula en tres ejes que se desarrollarán de manera transversal a lo largo del proceso de enseñanza aprendizaje, estos son la comunicación oral, la lectura y la escritura. Para este sector de aprendizaje, se plantea "que el lenguaje es la base de las demás áreas del saber y constituye en sí mismo una herramienta fundamental para la integración de las personas en una sociedad moderna, participativa y democrática” (Mineduc, 2009: 31). Este hecho se concreta a través del enriquecimiento y ampliación del lenguaje con que los estudiantes ingresan a los distintos establecimiento educacionales a través de la continua interacción y mejora de la comunicación oral y el acceso al lenguaje escrito por medio de la lectura y la escritura.

Complementa la presentación de este marco la explicitación de la propuesta metodológica que guiará el desarrollo de los diversos objetivos curriculares para el sector de aprendizaje. En tal sentido, se indica que la consecución de tales propósitos se concretará a través de un "enfoque comunicacional o comunicativo funcional, que considera el lenguaje como una herramienta eficaz de expresión, comunicación e interacción" (Mineduc, 2009: 31). En síntesis, el objetivo general del sector es el desarrollo de la competencia comunicativa que permita a los estudiantes interactuar de manera efectiva en diversas situaciones comunicativas a través de distintos textos y discursos.

Presentados estos lineamientos, resulta pertinente evidenciar la posible existencia de una tensión entre lo establecido en los mismos decretos y las posibilidades efectivas que brinda la formación inicial docente en las distintas instituciones de educación superior vinculadas a la formación de profesionales que enseñarán lengua materna, en términos del desarrollo y ampliación de estas competencias desde un enfoque como el que se propone, en el aula y otros espacios de interacción. Por otra parte, también es necesario revisar, entre otros aspectos, aquellos atingentes a las orientaciones y los espacios efectivos para el desarrollo de las competencias comunicativas. En este sentido, se puede sostener, por ejemplo, que se requieren precisar criterios y orientaciones curriculares que permitan a docentes desarrollar de manera óptima la enseñanza de la lengua en establecimientos con modalidad Formación Diferenciada Técnico Profesional, pues, de qué manera y en base a qué criterios se toman decisiones respecto a la implementación curricular en situaciones de enseñanza que ven reducido el tiempo destinado a la enseñanza de la asignatura. Asimismo, se puede sostener que, en la actualidad, la distribución del tiempo en relación a las actividades profesionales de los docentes, en la mayoría de los casos, se refiere a docencia en aula, en ausencia de espacios para la planificación y adecuación de la enseñanza; y si esos espacios existen, es fehaciente su utilización para responsabilidades administrativas. Así, en el escenario descrito, no es menor la complejidad existente al implementar didácticas de la escritura, oralidad y lectura que impliquen, por ejemplo, el trabajo personalizado junto a estudiantes, cuestión fundamental para afianzar las competencias en estos planos. Con todo, se espera que la Ley 20903 "Sistema de desarrollo profesional docente" contribuya en este último sentido. 


\subsection{PROGRAMAS DE ESTUDIO DE LA ENSEÑANZA MEDIA}

Los Programas de estudio de la Enseñanza Media (EM) materializan en unidades los aprendizajes para cada nivel de enseñanza. Desde primero a cuarto año medio, cada programa contempla cuatro unidades a desarrollar en el año académico, dos para el primer semestre y dos para el segundo semestre, que deben procurar el desarrollo de habilidades en los tres ejes ya enunciados: lectura, escritura y comunicación oral (Mineduc 2011a, 2011b, 2015a, 2015b). A continuación se sintetizan las principales habilidades para cada eje.

El eje de lectura contempla la revisión de distintos tipos de texto, de creciente complejidad y que promuevan el análisis crítico del entorno. Durante la lectura se prioriza la identificación de ideas y localización de información, la elaboración de resúmenes, el desarrollo de competencias de información, junto a la comprensión y dominio de nuevos conceptos que posibiliten la construcción de ideas propias y opiniones. Se propone, además, el uso sostenido de la biblioteca escolar CRA para fomentar el gusto por la lectura y el desarrollo de trabajos de investigación.

La escritura se propone a partir de prácticas que promuevan la elaboración de textos diversos en términos de extensión y complejidad, junto a la organización y presentación de información a través de esquemas o tablas. Se prioriza que la escritura desarrolle la presentación de ideas de manera coherente y clara, y el uso de vocabulario adecuado. Igualmente, debe promoverse el uso correcto de la gramática y convenciones ortográficas, el conocimiento y uso del lenguaje inclusivo.

Finalmente, el eje de comunicación oral procura que los estudiantes sean capaces de exponer en distintas situaciones comunicativas a través de la expresión de ideas y conocimientos de manera organizada; a ello se integra también el desarrollo de la argumentación. Es fundamental en este eje el desarrollo de la capacidad para escuchar. Se promueve, en este plano, el uso del lenguaje de manera precisa de acuerdo al nivel, el planteamiento de preguntas que posibiliten la expresión de dudas para superar dificultades en la comprensión, y la interacción con otras personas para intercambiar ideas, compartir puntos de vista y establecer acuerdos en relación a temas específicos.

Los elementos aquí descritos encuentran unos estadios de desarrollo para cada nivel en los Mapas de Progreso (Mineduc, 2008), instrumento que establece seis niveles de acuerdo a los 12 años de escolaridad y un séptimo nivel que se considera sobresaliente. Para el caso de la enseñanza media, los parámetros a observar corresponden al nivel 4, especialmente porque en él se señalan las habilidades esperadas que presentan los estudiantes al egresar del octavo año básico, es decir a su ingreso a la enseñanza media. Los niveles de progreso 5 y 6 corresponden a lo esperado de los estudiantes durante su formación de enseñanza media.

\section{ORIENTACIONES PARA EL DESARROLLO DE COMPETENCIAS COMUNICATIVAS EN EL PLANO ORAL Y ESCRITO EN LA ENSEÑANZA MEDIA}

\subsection{CARACTERIZACIÓN DEL LENGUAJE EMPLEADO EN LA ENSEÑANZA}

El lenguaje empleado en contextos educacionales corresponde a un registro académico. El registro académico presenta características que no siempre posibilitan el acceso a este tipo de discursos, en el plano escrito se trata de un lenguaje diferente al utilizado en la 
cotidianidad, que entre otras particularidades, se caracteriza por una alta planeación también en el plano oral-, una organización monológica y por el uso del lenguaje como reflexión (Halliday y Martin, 1993, cit. en Oteíza, 2006).

De acuerdo a lo anterior, algunas propuestas como las enmarcadas en la Lingüística Sistémica Funcional tienen entre sus propósitos proporcionar el acceso a los discursos de las ciencias a través de pedagogías de la alfabetización fundadas en el análisis del discurso (Martin y Rose, 2007) que propicien la reflexión sobre los contextos de producción y circulación de este tipo de discursos, sobre los textos que los constituyen, sobre las estructuras genéricas y sobre el lenguaje que los constituye (Moyano, 2013). De esta manera, es posible evidenciar junto a los estudiantes el funcionamiento del lenguaje en situaciones de comunicación concretas.

En relación a otras experiencias, se puede mencionar la reflexión a partir de los resultados de actividades desarrolladas en diversas aulas en el sur de Chile, cuyo propósito fue mejorar las habilidades comunicativas a través de la web social. Se señala que el rol que el currículum otorga de manera implícita al desarrollo de competencias comunicativas y su enseñanza pareciera llevarse a cabo de manera instrumental y fuera de los contextos en que ésta es efectivamente empleada (Cárdenas y Díaz, 2013). De esta manera, no es extraño advertir "prácticas educativas basadas en la estandarización, parcialización y desvinculación del lenguaje respecto de los fenómenos culturales a partir de los cuales se instancia" (Cárdenas y Díaz, 2013: 40). Junto a lo anterior, también es posible advertir en el ámbito nacional propuestas que con todo su aporte, se centran en el desarrollo de las habilidades asociadas a la lectura y la escritura definiendo estas en oposición al desarrollo del lenguaje oral, para el que se supone no se requieren una enseñanza formal como sí lo precisan la lectura y escritura (Velásquez et al., 2006). En tal sentido, las experiencias mencionadas indican que es central el rol que se otorga al lenguaje dentro del proceso de enseñanza, como también su análisis a partir de situaciones comunicativas concretas que contemplen desde la participación de estudiantes en situaciones comunicativas cotidianas, hasta aquellas que implican un conocimiento y preparación acorde a registros específicos, como los académicos.

En este contexto, el desarrollo de competencias comunicativas en el plano oral y escrito en la enseñanza media debiera enmarcarse en una propuesta metodológica general que contemple situaciones concretas de comunicación, es decir, se debe integrar un análisis de las características del contexto en que los géneros discursivos son producidos, junto al análisis de las características de los mismos: propósitos sociales, modos de organización y recursos lingüísticos que los realizan.

Como especifica Cassany (2008), el interés por estudiar cada género está en que, al conocer cómo es y cómo funciona, se puede mejorar su enseñanza y aprendizaje, con lo cual aprender a utilizar un género es aprender a desarrollar las prácticas profesionales que se desarrollan en él. Así, el análisis propuesto debe considerar los aspectos socioculturales y contextuales en los cuales se incluyan los elementos básicos del acto comunicativo; aspectos discursivos referidos a la organización del discurso en el plano supraoracional, los apartados y subapartados, los párrafos, las secuencias discursivas o los códigos no verbales, y los aspectos gramaticales y léxicos que consideran la elección de las palabras y estructuras (Cassany, 2008).

A modo de síntesis, resulta prioritario disponer de enfoques didácticos y estrategias metodológicas que permitan adecuar la enseñanza y desarrollo de las competencias 
comunicativas a los distintos contextos de enseñanza, pues tal y como sostiene Marta Marín "los cambios didácticos no se dan en forma independiente de los modelos pedagógicos" (2008: 26).

\subsection{LA LENGUA ORAL FORMAL: CARACTERÍSTICAS LINGÜÍSTICAS Y DISCURSIVAS}

En el aula se sigue operando con la concepción de que el ámbito de la oralidad y la escritura corresponden a manifestaciones dicotómicas que consideran, por un lado, la conversación espontánea como el género prototípico de la oralidad en contraposición a la escritura académica como la manifestación prototípica de la escritura "sin tener en cuenta las posiciones intermedias" (Castellà y Vilà i Santasusana, 2005: 25). Parodi (2003) también establece esta dicotomía entre lo oral y lo escrito cuando se refiere al texto oral como una variedad que contiene "una relativamente escasa variedad léxica y una sintaxis más bien suelta y abierta, es decir, no denota un alto grado de cohesión", seguido de la norma escrita que "en su punto extremo es caracterizada como formal, altamente planificada y artificial" (2003: 36).

No obstante lo anterior, en la enseñanza de la oralidad en la escuela se debiera considerar que las características de la lengua oral formal comparten muchas de las particularidades de la escritura y que, en este sentido, viene a constituir un género intermedio entre la oralidad espontánea y la escritura académica (Castellà y Vilà i Santasusana, 2005) que requiere sistematizar su enseñanza tal como se pudiera plantear la enseñanza de la escritura. Bajo esta concepción, es preciso entender que esta dicotomía inicial se atenúa al proponer una relación gradual desde la lengua oral informal como género espontáneo, la lengua oral formal como género intermedio y la escritura como un registro más planificado y complejo. Así, es preciso que el registro oral formal también sea considerado como una habilidad que se debe enseñar.

Por mucho tiempo se consideró que la expresión oral no necesitaba instrucción, pues se trataba de una habilidad aprendida de manera natural. Considerando ahora el hecho de que la oralidad también se desarrolla en ambientes formales y necesita de una estricta planificación, es necesario instruir a los estudiantes de manera que puedan ejecutar correctamente los textos orales planificados, pues en la oralidad también sería posible distinguir entre géneros discursivos primarios (simples) y géneros discusivos secundarios (complejos) (Bajtín, 2008).

Aun cuando Bajtín (2008) entiende que los géneros secundarios "surgen en condiciones de la comunicación cultural más compleja, relativamente más desarrollada y organizada, principalmente escrita" (Bajtín, 2008: 247), algunas situaciones orales se constituyen en instancias que requieren planificación y reflexión previa al igual que en los géneros secundarios (Solè, 2007); por lo cual, la propuesta de Castellà y Vilà i Santasusana (2005) incorpora la noción de género intermedio para dar cuenta de las situaciones orales que se alejan de la espontaneidad de la conversación coloquial y se acercan a la formalidad y la planificación de la escritura. Estos géneros orales intermedios comparten, por cierto, rasgos de la conversación coloquial, por cuanto requieren de la presencia de los interlocutores y hacen uso de elementos paraverbales y no verbales, por ejemplo; pero también adoptan características propias de las convenciones del texto escrito en cuanto a que el discurso se desarrolla en torno a un tema especializado, necesita una planificación previa y sigue la estructura propia de la exposición (Tabla 1). 
Tabla 1. Comparación de los rasgos de la lengua oral formal con la escritura

\begin{tabular}{|l|l|}
\hline $\begin{array}{c}\text { RASGOS EN LOS QUE LA LENGUA ORAL } \\
\text { FORMAL SE SITUA EN POSICIÓN } \\
\text { INTERMEDIA ENTRE LA LENGUA ORAL } \\
\text { FORMAL Y LA ESCRITURA }\end{array}$ & $\begin{array}{c}\text { RASGOS QUE LA LENGUA ORAL } \\
\text { FORMAL COMPARTE CON LA } \\
\text { ESCRITURA }\end{array}$ \\
\hline $\begin{array}{l}\text { Interacción media emisor- receptor: comunica- } \\
\text { ción relativamente unidireccional. }\end{array}$ & $\begin{array}{l}\text { Carácter no universal que requiere aprendizaje } \\
\text { generalmente académico }\end{array}$ \\
\hline $\begin{array}{l}\text { Relativa distancia emotiva: espacio más o me- } \\
\text { nos reglado. }\end{array}$ & Generalmente monologada. \\
\hline Información contextual relativamente explicita. & Formal. \\
\hline Estructura textual bastante estereotipada. & De tema especializado y planificada. \\
\hline Selección relativa del léxico: variación media. & Informativa y de tono relativamente objetivo. \\
\hline $\begin{array}{l}\text { Presencia media de elementos deícticos. Es más } \\
\text { informativa que interactiva. }\end{array}$ & $\begin{array}{l}\text { Corrección normativa y uso de la variedad es- } \\
\text { tándar. }\end{array}$ \\
\hline $\begin{array}{l}\text { Estructuras sintácticas propias de los estilos no- } \\
\text { minal y verbal }\end{array}$ & \multicolumn{2}{|l}{} \\
\hline
\end{tabular}

Adaptado de Castellà y Vilà i Santasusana (2005)

A este respecto, Castellà y Vilà i Santasusana (2005) sugieren caracterizar la lengua oral formal en relación a sus rasgos contextuales, discursivos y lingüísticos, tal como se aprecia en la Tabla 1. En relación al contexto, la lengua oral formal está mediada por el aprendizaje escolar y comparte con la conversación prototípica el hecho de que su producción se produce en tiempo real mediante el canal acústico en un contexto situacional compartido entre emisor y receptor. Aunque en la lengua oral formal, a diferencia de la conversación, se supone una intervención unicontrolada, en la cual los turnos de palabra están limitados y son eventualmente gestionados por el emisor. Los rasgos discursivos de la lengua oral formal aluden a un discurso formal que generalmente es expositivo y monologado y corresponden a discursos informativos muy planificados que requieren una organización cuidadosa del tema de la exposición y la estructura de la misma.

Para Vilà i Santasusana (2005), la variedad oral formal, así descrita, se manifiesta en el discurso explicativo que, por desarrollarse típicamente en el ámbito de la enseñanza y tener la versatilidad de adaptarse a las múltiples formas de situaciones comunicativas que se generan en el aula, debiera ser el discurso privilegiado en la didáctica de la lengua oral formal. Es así como la enseñanza debe apuntar a las convenciones del discurso oral formal, pero también a desarrollar las estrategias que permitan estructurar un tipo de discurso explicativo en cuyo caso estas estrategias tienen relación con explicitar la estructura del discurso, contextualizar el discurso y regular la densidad informativa del mismo (Vilà $\mathrm{i}$ Santasusana, 2005). Por su parte, Solè (2007), al referirse a la lengua oral formal en el ámbito académico, especifica que ésta asume una estructura expositiva que, de manera general, supone la variedad explicativa y la argumentativa. 
Otro aspecto a desarrollar en el texto oral formal es la modalización (Grau, 2005), entendida como las marcas que el emisor deja en el mensaje mediante recursos lingüísticos y prosódicos en relación a su compromiso con el tema desarrollado y a los grados de certeza con que se exponen los datos. A este respecto, las dificultades de los estudiantes se presentan al valorar cuándo es adecuado introducir opiniones personales versus exposición de enunciados de una manera distanciada, pues es natural que no conozcan los elementos lingüísticos que les permitirían distanciarse del discurso. Tampoco están conscientes de la necesidad de usar fuentes de información adecuadas, no saben regular el grado de certeza con el cual exponen los contenidos, y en la dinámica de la interacción que se puede producir en una exposición, sobre todo, si se considera una fase final de preguntas, tienen problemas para expresar con respeto el desacuerdo y responder a situaciones más o menos conflictivas (Grau, 2005).

\subsection{LA LENGUA ESCRITA: CARACTERÍSTICAS LINGÜÍSTICAS Y DISCURSIVAS}

Es un hecho que la adquisición de la lengua escrita requiere de un proceso de enseñanza aprendizaje sistematizado, porque "a pesar de que la lengua escrita está presente en el entorno cotidiano, el aprendizaje del código exige un adiestramiento y una preparación específica" (Calsamiglia y Tusón, 1999: 66). Este adiestramiento no termina, por cierto, con la adquisición del código sino que debe avanzar hacia la práctica constante de la escritura en contextos reales, por cuanto,

cada uso lingüístico, cada actividad de composición, es un acto contextualizado que tiene lugar en unas circunstancias temporales y espaciales (...) el lenguaje no es un código abstracto ni desvinculado de sus usuarios, sino que surge y se utiliza en una comunidad de hablantes que comparte una misma concepción del mundo (Cassany, 2000: 27).

Es importante destacar lo que algunos autores han descrito como el potencial epistémico de la escritura, bien como una de las principales dificultades asociadas a la adquisición de esta competencia (Carlino, 2004), bien como uno de los elementos fundamentales asociados al dominio y práctica de la escritura (Marín, 2015). En definitiva, se debe considerar que el dominio y ejercicio de la escritura es también una forma de crear conocimiento, y de aprender la escritura misma y los conocimientos de todas las otras disciplinas del currículum escolar.

Los registros escritos se caracterizan por desarrollarse en una situación de enunciación en la cual la relación entre el emisor y sus lectores se produce de forma diferida, por cuanto no se ubican simultáneamente en el mismo momento ni en el mismo lugar. Esta relación afecta las características propias de la modalidad escrita en cuanto, por ejemplo, debe suplir la deixis del texto oral y contener todos los elementos lingüísticos y discursivos que guíen de forma adecuada la interpretación del lector. Además, la modalidad escrita, aun cuando puede contemplar grados de informalidad, se caracteriza mayoritariamente por el uso de registros formales y de la variedad estándar de la lengua.

La complejidad de la escritura radica primordialmente en el carácter monogestionado que obliga al escritor a desarrollar un proceso reflexivo, en ausencia del receptor, cuyo producto sea un texto que combine de forma adecuada la profundidad en el tratamiento de 
la información, la elección de las estructuras gramaticales y la sintaxis, y el uso preciso del léxico. Junto con esto, la jerarquización de las ideas implica un conocimiento acerca de las convenciones del texto escrito en su dimensión formal y material. Los textos escritos "son sistemas complejos de unidades lingüísticas de diferentes niveles (párrafos, oraciones, sintagmas, palabras) y de reglas o criterios de organización de las mismas (introduccióndesarrollo-conclusión, tesis-argumento, causa-consecuencia, coordinación sujeto-verbo)" (Cassany, 2000:32) ${ }^{1}$.

En relación a los recursos lingüísticos, el texto escrito se caracteriza por una complejidad gramatical en la cual predomina el uso de vocabulario especializado y de nominalizaciones (Castellà y Vilà i Santasusana, 2005). Se entiende la nominalización como el recurso por el cual se transforman verbos y adjetivos en sustantivos, "de esta manera se tratan acciones, procesos, etc., como objetos separados de la participación de agente humano y se generan términos técnicos para nombrarlos" (Teberosky, 2007: 35). Este estilo nominal implica a su vez el uso de adjetivos, de complementos nominales, de sintagmas preposicionales en función de complementos del nombre y de oraciones de relativo.

Sería necesario también considerar en la escritura la modalización, tal como fue explicada en relación al texto oral formal. En el texto escrito, generalmente, el emisor toma distancia del contenido expuesto y construye un texto impersonal y con marcas de objetividad en el cual además no hay marcas explícitas de implicación del destinatario del texto. En relación al tratamiento de la información, normalmente, esta se presenta exenta de juicios de valor y con uso de recursos lingüísticos que implican un alto grado de certeza. Junto con esto es posible validar la información transmitida considerando la mención de las fuentes utilizadas.

Por otro lado, la enseñanza de la lengua escrita, al igual que lo planteado a propósito de la lengua oral formal, debe enfocarse desde el concepto de género para entender que cada acto comunicativo está definido por un propósito socialmente reconocido y "los textos que producen un hablante (...) se insertan en la tradición discursiva que ha desarrollado su comunidad lingüística a lo largo de su existencia" (Cassany, 2000: 33). De este modo,

los textos generados por una comunidad se reparten entre distintos contextos sociales o esferas comunicativas (...) cada una de estas esferas dispone de su propia tradición discursiva, que contiene convenciones aceptadas por la comunidad de hablantes, más o menos explícitas y conscientes, que afectan al contenido (temas, estructura) y a la forma (registro, tono fraseología) de los discursos. Denominamos género a cada una de estas esferas sociales de comunicación, que puede contener tipos de textos más concretos (Cassany, 2000: 33).

No hay que olvidar que Bajtín (2008) define los géneros secundarios en relación precisamente con su carácter escrito. En el contexto de este trabajo, un género prototípico de la escritura lo conformaría el texto académico o el texto escrito producido en el aula, por tanto, es preciso acudir al reconocimiento de este género en particular así como a los recursos discursivos y gramaticales que posibilitan su redacción. En resumen, "aprender a escribir significa aprender a dominar cada uno de los géneros verbales para conseguir los objetivos deseados" (Cassany, 2000: 35).

Cursiva en original. 


\subsection{DIDÁCTICA DE LA ENSEÑANZA DE LA LENGUA ORAL FORMAL}

En base a los conceptos expuestos en la caracterización del discurso oral formal, se proponen los siguientes lineamientos para la enseñanza y práctica del discurso oral formal en secundaria. Primero, es necesario dedicar tiempo en el aula para guiar la preparación del discurso oral formal si se le considera un género intermedio que requiere planificación (Castellà y Vilà i Santasusana, 2005; Solè, 2007). Después del proceso de documentación de un tema específico, que el estudiante podría realizar de forma autónoma o guiada, el profesor debería monitorear la planificación de la exposición en diferentes etapas. Lo primero sería decidir la estructura de la disertación (Briz, 2008; Vilà i Santasusana, 2005), es decir, el esquema del desarrollo que es la parte medular de la exposición. En esta parte, se sugiere ordenar los datos del cuerpo de la presentación en un esquema tripartito que considere una secuencia lógica que el oyente pueda seguir.

Se pueden ensayar distintos esquemas que se ajusten a la naturaleza del tema expuesto (Briz, 2008). Por ejemplo, se puede trabajar con un esquema en el cual se ordenen los hechos cronológicamente desde el pasado hasta el presente y proyecciones que apunten al futuro. Se podría también identificar la causa de un fenómeno, el desarrollo de este y los efectos que provoca. En el caso de trabajar con un problema específico, sería aceptable comenzar con una descripción de la situación, para identificar el problema y terminar con una solución o una propuesta de solución. También se puede identificar un objeto, ofrecer una descripción del mismo y explicar cómo funciona. Quizás sea necesario identificar un conjunto, dividirlo en partes, explicar sus relaciones. Además, se puede considerar un esquema deductivo que abarque en tres partes de lo general a lo particular o el ejemplo, etc. Las posibilidades son muchas y el estudiante debe explorarlas para encontrar la mejor opción que le permita ordenar de forma lógica el contenido de su presentación.

Una vez consensuado el esquema, el alumno debe aportar los datos relevantes que permitan aplicar esta pauta a su tema específico. Resulta útil en esta etapa completar las etiquetas del esquema con palabras o conceptos claves, a partir de los cuales es posible ir ampliando de forma ordenada la información de que se dispone. En este punto es necesario poner atención a la elección del léxico que debe obedecer a criterios de formalidad y precisión. Junto con esto, el estudiante debe cuidar que la redacción de las ideas se haga con oraciones cortas de sintaxis regular para evitar la ambigüedad (Briz, 2008). Salvo que se trate de secuencias narrativas que contemplen alusiones a hechos pasados, las formas verbales para redactar el desarrollo de la exposición deberían ser predominantemente el presente simple, el presente perfecto y el futuro simple de indicativo. Es posible considerar formas perifrásticas tanto en el presente como en el futuro, pues estas están más relacionadas con la oralidad que con la escritura; sin embargo, las formas simples le otorgan un mayor grado de formalidad al texto.

En esta etapa de la planificación, también se hace necesario consolidar las elecciones a nivel de modalidad (Grau, 2005), de modo que si el estudiante ha decidido o se le ha solicitado que exponga un texto objetivo, con altos grados de certeza, exento de valoraciones y con adecuado respaldo teórico, deberá cuidar de borrar las marcas de primera persona en el texto para distanciarse del contenido al mismo tiempo que prefiere formas verbales en tercera persona o con marcas de impersonalidad. Debe cuidar las elecciones de finitos modales y adjuntos modales que expresen certeza y evitar el léxico que apunte a posiciones valóricas. 
Todavía hay otros recursos lingüísticos sobre los cuales poner atención, como escoger adecuadamente los conectores, ya sea aquellos que sirven para ir conectando de forma apropiada las ideas, como los marcadores de orden que facilitan a los receptores del discurso seguir la exposición. Junto con esto se pueden ejercitar y aplicar estrategias que apunten a facilitar la comprensión de los oyentes como el uso de ejemplos, reformulaciones, repeticiones, énfasis, etc.

Una vez que el estudiante haya planificado, con la ayuda del profesor, el desarrollo de su exposición, podrá proyectar el contenido del exordio y el epílogo para cumplir con los propósitos para cada una de estas fases (Aristóteles, 2011; Vilà i Santasusana, 2005). A saber, saludar, presentar el tema, captar la atención del público, compartir el esquema de la presentación en el exordio, y resumir, concluir, enfatizar y agradecer en el epílogo.

La revisión de esta planificación en sus diferentes etapas puede ser guiada por el profesor, pero también resultaría beneficioso trabajar con evaluaciones y discusiones entre pares en relación a los avances de cada uno. Superadas las etapas de planificación propuestas, el estudiante estaría en condiciones de exponer ante sus compañeros y el profesor para lo cual tendría que gestionar los aspectos paraverbales y no verbales que son imprescindibles en la comunicación oral (Briz, 2008; Grau i Taurruel y Vilà i Santasusana, 2005). Esta exposición podría, además, estar apoyada por una presentación multimedia que también debe ser planeada cuidadosamente (Solè, 2007).

\subsection{DIDÁCTICA DE LA ENSEÑANZA DE LA LENGUA ESCRITA}

En la enseñanza de la escritura es necesario relevar la noción de la escritura como una actividad social y cultural compleja que se relaciona con las prácticas discursivas en las cuales se produce. Así, se parte del texto "definido como unidad real de comunicación a través de la cual se negocia significado" (Moyano, 2013: 35). Se trata, por tanto, de orientar el reconocimiento de las convenciones discursivas de los géneros mediante el desarrollo de una didáctica basada en la enseñanza de los mismos con "énfasis en el contenido, la estructura de los textos y la realización gramatical” (Amaya, 2013: 100).

La propuesta didáctica que se comenta a continuación contempla los postulados de la escuela de Sydney y su pedagogía basada en el reconocimiento y enseñanza de los géneros discursivos. Esta escuela plantea la práctica de la escritura en tres etapas que consideran primero el análisis de las características de un género real: la deconstrucción del género. En una segunda fase se procede a la construcción conjunta para derivar en un tercer momento de construcción independiente, en ambas etapas se implica la escritura guiada e individual. Para entender este proceso, resulta útil acudir a experiencias de aplicación en el aula como las de Moyano (2007), y Roldán, Cárdenas y Casanova (2013), que aplican el modelo en contextos iniciales de enseñanza terciaria a partir de las competencias que los estudiantes poseen al inicio de los estudios de pregrado. Estela Moyano propone, tal como se aprecia en el Cuadro 1, momentos para la edición y evaluación de las producciones escritas de los estudiantes.

La tarea de escritura comienza así con la etapa de deconstrucción que consiste en "introducir a los estudiantes en un ejemplo de texto perteneciente al género en estudio" (Moyano, 2007: 578). El propósito es que los estudiantes puedan entender a partir de un texto ejemplar o varios textos del mismo género cómo está organizado el género sometido a análisis en relación a la producción de significados y el cumplimiento del propósito social y comunicativo perseguido. 
Cuadro 1. Propuesta didáctica para el desarrollo de la escritura centrada en los géneros

\begin{tabular}{|c|c|c|c|}
\hline \multirow{9}{*}{$\begin{array}{l}\text { Situación social en } \\
\text { la que surge el texto } \\
\text { Determinación del } \\
\text { contexto }\end{array}$} & \multirow{2}{*}{\multicolumn{2}{|c|}{ Deconstrucción del género }} & Lectura conjunta \\
\hline & & & Lectura en pequeños grupos \\
\hline & \multirow{4}{*}{ Diseño del texto } & \multirow{4}{*}{$\begin{array}{l}\text { Construcción de } \\
\text { ejemplares genéricos }\end{array}$} & \\
\hline & & & Escritura conjunta \\
\hline & & & Escritura en pequeños grupos \\
\hline & & & Escritura individual \\
\hline & \multirow{3}{*}{\multicolumn{2}{|c|}{$\begin{array}{l}\text { Edición de las producciones por } \\
\text { parte de los estudiantes }\end{array}$}} & Evaluación conjunta \\
\hline & & & Evaluación en pequeños grupos \\
\hline & & & Evaluación individual \\
\hline
\end{tabular}

Fuente: Moyano (2007)

La etapa de la deconstrucción del género posibilita la reflexión en torno a las condiciones del contexto y los elementos de la situación retórica que condicionan las elecciones lingüísticas en el texto relacionadas tanto con las condiciones del contexto de producción como con las condiciones del contexto de recepción y circulación de los textos. Con ello se explican desde una perspectiva lingüística tanto las diferencias como los aspectos recurrentes -tipos relativamente estables, como señala Bajtín (2008)-, y aún necesarios, en los textos que se producen (Eggins y Martin, 2000).

La etapa de construcción o escritura puede transitar desde la construcción conjunta (con el profesor o con un grupo de pares) a la construcción o escritura individual. Se trata de ajustar el contenido a la estructura relevada en el análisis previo, para lo cual es necesario que el estudiante ordene y jerarquice la información y luego aplique de forma eficiente las particularidades del género.

Finalmente, en la edición de los textos se monitorea de qué modo la producción se ajusta a las características del género relevadas en las etapas previas. La reflexión sobre la construcción del texto tiene por objeto además evaluar la "realización lingüística, la selección y organización de la información, sus condiciones de textura, los aspectos léxicogramaticales, sus aspectos gráficos, etc.” (Moyano, 2007: 582).

Así descrito el método, es factible también abordar la escritura como un proceso complejo y recursivo que necesita intervención, guía, edición y evaluación en el desarrollo del proceso mismo. A menudo la práctica en el aula descuida la producción individual, cuando se solicitan tareas de escritura grupal, y descuida el proceso, cuando la corrección se ubica una vez concluido éste y se corrige solo la versión última del escrito.

Sin embargo, es preciso visibilizar el proceso que subyace a la escritura de un texto. Este proceso, según el ya conocido modelo de Scardamalia y Bereiter (1992), requiere una gestión del tiempo que demanda tanto la tarea de composición según la dificultad del texto a escribir como la ejecución de las fases de la composición. Estas fases partirían, (completando la propuesta de los autores) por el adecuado procesamiento de la información e identificación de fuentes a consultar, etapa que se completa con el registro de las fuentes y los datos e información consultados en ellas. A este proceso seguiría la jerarquización de 
la información y la planificación a modo de esquema de los puntos que se van a desarrollar por escrito. Esto conduce a la tarea misma de escritura que deriva en sucesivos borradores que deben ser revisados de modo de integrar la revisión al proceso de composición para que no quede relegada, como en muchos casos, a la fase evaluativa final de la versión final del escrito. Se pone de relieve, entonces, que la revisión es parte del proceso de composición y puede ser abordada por el docente o por el propio estudiante, quien con la guía del profesor adquiere las herramientas metodológicas que le permitan evaluar el progreso de su propio texto. La revisión constante y la planificación previa derivarán finalmente en una versión mejorada que puede ser sometida a evaluación en conjunto con el proceso mismo.

\section{CONCLUSIONES}

La revisión y actualización aquí presentada expuso alcances sobre el trabajo de Burgos (1976) realizado hace ya 40 años, en conmemoración del aniversario de la aparición del primer número de la revista "Estudios pedagógicos". Se siguió un itinerario muy similar al planteado en el original para el tratamiento del tema; se han incluido los lineamientos prescritos por el Mineduc para el desarrollo de las habilidades en la actual asignatura Lenguaje y Comunicación, y antecedentes que pueden complementar la didáctica de la enseñanza de la lengua en sus planos oral y escrito.

El desarrollo de la competencia comunicativa es un área absolutamente vigente en materia de investigación. Diversas son las publicaciones a nivel nacional, latinoamericano y mundial que dan cuenta de una sostenida preocupación por la inequidad en el acceso a los discursos académicos, profesionales y científicos, y en tal sentido conviene revisar de manera crítica aquellas perspectivas que puedan complementar el trabajo en el aula desde la perspectiva de la alfabetización académica, por cuanto ella representa el desafío de una formación constante cuyo propósito es posibilitar la adscripción a distintas comunidades discursivas a través del aprendizaje de sus modos de ser en y a través del discurso que las caracteriza: distintos niveles de formación exigen el desarrollo de las competencias comunicativas específicas a esos niveles.

En general, los alcances en relación a las propuestas didácticas relevadas en este trabajo, tanto para la oralidad como para la escritura, coinciden en abordar estas competencias desde una perspectiva de género, lo que conlleva el análisis de éstos en conjunto con los estudiantes, con el propósito de advertir la forma en que el lenguaje construye significado, relevando, entre otros aspectos, la estructura de los textos, sus patrones gramaticales y el contenido (Moyano, 2007).

Se puede sostener que, como complemento al despliegue didáctico propuesto, es relevante profundizar en usos efectivos para los denominados recursos de aprendizaje de los que disponen los estudiantes en las aulas y en los establecimientos educativos en general. Quizá una posible respuesta se encuentre en la sistematización de prácticas docentes, su análisis y el establecimiento de orientaciones a partir de ellas, para complementar el uso que se hace de las tecnologías de la información y la comunicación, y de las bibliotecas que se encuentran en los establecimientos educacionales, entre otros recursos. Lo anterior se puede complementar con el estudio de las concepciones docentes que guían estas prácticas, a veces, de manera no explícita, para advertir qué media la decisión del profesorado, y también de los estudiantes, al usar estos recursos. 


\section{REFERENCIAS BIBLIOGRÁFICAS}

Amaya, O. (2013). Apropiación de contenidos en comunidades de pensamiento científico: prácticas discursivas y estrategias metacognitivas. En E. Moyano (Coord.), Aprender ciencias y humanidades: una cuestión de lectura y escritura. Aportes para la construcción de un programa de inclusión social a través de la educación lingüística (pp. 79-107). Buenos Aires: Universidad Nacional de General Sarmiento.

Aristóteles (2011). Arte poética. Arte retórica (4ta ed.). México: Editorial Porrúa.

Bajtín, M. (2008). El problema de los géneros discursivos. Estética de la creación verbal (2da ed. argentina revisada, pp. 245-290). Buenos Aires: Siglo XXI Editores, S.A.

Briz, A. (2008). Saber hablar. Buenos Aires: Aguilar.

Burgos, J. (1976). Didáctica de la expresión oral y escrita en el liceo. Estudios pedagógicos, 1, 25-35.

Calsamiglia, H. y Tuson, A. (2007). El discurso escrito. Las cosas del decir. Manual de análisis del discurso (2a ed., pp. 15-58). Barcelona: Ariel.

Cárdenas, C. y Díaz, C. (2013). Jóvenes, tecnología y competencias sociocomunicativas: Educación para una relación necesaria. En L. Cárcamo, E. Scheihing y C. Cárdenas (Eds.), Didáctica 2.o. La web social en el aula (pp. 39-50). Valdivia: Ediciones Kelluwen.

Carlino, P. (2004). El proceso de escritura académica: cuatro dificultades de la enseñanza universitaria. Revista Educere. Investigación, 8(26), 321-327.

Castellà, J.M. y Vilà i Santasusana, M. (2005). La lengua oral formal: características lingüísticas y discursivas. En M. Vilà i Santasusana (Coord.), El discurso oral formal. Contenidos de aprendizaje y secuencias didácticas (pp. 25-36) Barcelona: Graó.

Cassany, D. (2000). Construir la escritura. Barcelona: Paidós.

Cassany, D. (2008). Taller de textos. Leer, escribir y comentar en el aula. Barcelona: Paidós.

Eggins, S. y Martin, J. (2000). Géneros y registros del discurso. En T. Van Dijk (Comp.), Estudios sobre el discurso I. Una introducción multidisciplinaria. El discurso como estructura y proceso (pp. 335-371). Barcelona: Editorial Gedisa.

Grau, M. (2005). La modalización: adecuación del texto a los parámetros de la situación comunicativa. En M. Vilà i Santasusana (Coord.), El discurso oral formal. Contenidos de aprendizaje y secuencias didácticas (pp. 77-88). Barcelona: Graó.

Grau i Taurruel, M. y Vilà i Santasusana, M. (2005). La competencia prosódica y la comunicación no verbal. En M. Vilà i Santasusana (Coord.), El discurso oral formal. Contenidos de aprendizaje y secuencias didácticas (pp. 89-100). Barcelona: Graó.

Marín, M. (2015). Escribir textos científicos y académicos. Buenos Aires: Fondo de Cultura Económica.

(2008). Lingüística y enseñanza de la lengua (2da ed. actualizada). Buenos Aires: Aique grupo editor.

Martin, J. y Rose, D. (2007). Working with discourse (2nd ed.). Londres: Continuum.

MINEDUC. (sf). Mapas de Progreso del Aprendizaje. Lectura. Santiago de Chile: Autor.

(2015a). Lenguaje y comunicación. Programa de estudio tercer año medio. Santiago de Chile: Autor.

. (2015b). Lenguaje y comunicación. Programa de estudio cuarto año medio. Santiago de Chile: Autor.

. (2011a). Lenguaje y comunicación. Programa de estudio primer año medio. Santiago de Chile: Autor.

. (2011b). Lenguaje y comunicación. Programa de estudio segundo año medio. Santiago de Chile: Autor.

. (2009). Objetivos Fundamentales y Contenidos Mínimos Obligatorios de la Educación Básica y Media. Actualización 2009. Santiago de Chile: Autor.

. (2008). Mapas de Progreso del Aprendizaje. Producción de Textos Escritos. Santiago de 
Chile: Autor.

Moyano, E. (2013). Introducción. Ciencia, discurso y participación social: la escuela como agente de redistribución del poder. En E. Moyano (Coord.), Aprender ciencias y humanidades: una cuestión de lectura y escritura. Aportes para la construcción de un programa de inclusión social a través de la educación lingüística (pp. 17-27). Buenos Aires: Universidad Nacional de General Sarmiento.

. (2007). Enseñanza de habilidades discursivas en español en contexto pre-universitario: Una aproximación desde la LSF. Revista Signos, 40(65), 573-608.

Oteíza, T. (2006). Situando los textos escolares en una práctica social. El discurso pedagógico de la historia (pp. 51-79). Santiago: Frasis.

Parodi, G. (2003). Relaciones entre lectura y escritura: una perspectiva cognitiva discursiva. Bases teóricas y antecedentes empíricos. Valparaíso: Ediciones Universitarias de Valparaíso.

Roldán, Y., Cárdenas, C. y Casanova, R. (2013). Teoría y práctica de la escritura académica. Guía para la producción y evaluación de un Informe Académico. Valdivia: Ediciones Universidad Austral de Chile, Publicaciones de la Facultad de Filosofía y Humanidades.

Solè, I. (2007). La exposición pública del trabajo académico: del texto para ser leído al texto oral. En M. Castelló (Coord.), Escribir y comunicarse en contextos científicos académicos. Conocimientos y estrategias (pp. 113-135). Barcelona: Graó.

Scardamalia, M. y C. Bereiter. (1992). Dos modelos explicativos de los procesos de composición escrita. Infancia y aprendizaje, (58), 43-64.

Teberosky, A. (2007). El texto académico. En M. Castelló (Coord.) Escribir y comunicarse en contextos científicos y académicos (pp. 17-46). Barcelona: Graó.

Velásquez, M., Peronard, M., Alonzo, T., Ibáñez, R. y Órdenes, J. (2006). Guiones metodológicos para desarrollar estrategias de comprensión y producción de textos escritos. Valparaíso: Ediciones Universitarias de Valparaíso.

Vilà i Santasusana, M. (2005). El discurso explicativo oral: estrategias comunicativas. En M. Vilà i Santasusana (Coord.), El discurso oral formal. Contenidos de aprendizaje y secuencias didácticas (pp. 37-56). Barcelona: Graó. 
